\title{
A Mechanochemical Zinc-Mediated Barbier-Type Allylation Reaction under Ball-Milling Conditions
}

\author{
JieXiang Yin,${ }^{\dagger}$ Roderick T. Stark, ${ }^{\dagger}$ Ian A. Fallis, ${ }^{\dagger}$ and Duncan L. Browne, ${ }^{*}+t$ \\ ${ }^{\dagger}$ School of Chemistry, Cardiff University, Main Building, Park Place, Cardiff, CF10 3AT, U.K. \\ ¥Current Address: UCL School of Pharmacy, 29-39 Brunswick Square, London, WC1N 1AX, U.K. \\ *E-mail: duncan.browne@ucl.ac.uk
}

Supporting Information

\begin{abstract}
A Ball-milling enabled zinc-mediated Barbier-type allylation reaction is reported. Notably runnning the reaction in this manner renders it effective irrespective of the initial morphology of the zinc metal. The process is operationally simple, does not require inert atmospheres or dry solvents and is reported on a range of aldehyde and ketone substrates, a gram scale process is demonstrated.
\end{abstract}

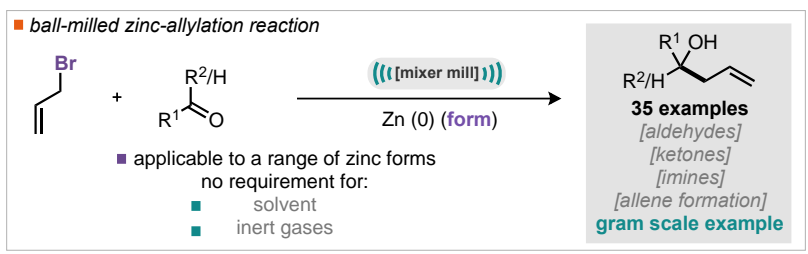

\section{INTRODUCTION}

The Barbier reaction features the use of base metals, in their zero-valant form, to couple alkyl halides with carbonyl compounds. ${ }^{1}$ Since the seminal publication by Barbier in $1899,{ }^{2}$ the use of a variety of metals has been reported, such as, zinc, ${ }^{3 \mathrm{a}}$ magnesium, ${ }^{3 \mathrm{~b}}$ samarium, ${ }^{3 \mathrm{c}}$ aluminium, ${ }^{3 \mathrm{~d}}$ indium, ${ }^{3 \mathrm{e}}$ cadmium, ${ }^{3 \mathrm{f}}$ antimony, ${ }^{3 \mathrm{~g}}$ lead, ${ }^{3 \mathrm{~h}}$ tin, ${ }^{3 \mathrm{i}}$ bismuth $^{3 \mathrm{j}}$ and manganese ${ }^{3 \mathrm{k}}$ as well as asymmetric processes ${ }^{4}$ and even aqueous media variants. ${ }^{5}$ Perhaps the most studied Barbier-type reaction is the allylation of carbonyl groups to deliver versatile homoallylic alcohol products. ${ }^{1}$ A common feature of these processes is the requirement of a base metal, whose physical form, solubility and oxide surface layer can lead to variable outcomes. Indeed, the use of chemical additives to circumvent these issues has been well studied (A, Scheme 1). ${ }^{6}$

Recently, we and others have been investigating the use of mechanochemistry ${ }^{7}$ to impart impact and shear forces onto reactants via ball-milling and thus mechanically activate, rather than chemically activate, zinc metal in a variety of forms. ${ }^{8}$ In addition to the ability to mechanically activating metals, ball-milling often requires the use of no or very little solvent (for the reaction portion at least) and can lead to interesting reaction profiles and reduced sensitivity of reactions to oxygen and/or water. ${ }^{9}$ To date, we have outcomes, such as; reduced reaction times, alternate selectivity demonstrated, in the context of zinc-mediated transformations, that a ball-milling approach can deliver an improved protocol for the Negishi crosscoupling and Reformatsky reactions (B, Scheme 1). Under these processes it was found that the reactions were operable irrespective of the morphology of the zinc starting material. Thus simply adding the appropriate reagents to the grinding jar under an air atmosphere followed by closing the jar and milling on commercially available equipment led to the de- sired products in good yields and across a range of substrates. $^{8 \mathrm{ab}}$

Herein we report the application of ball-milling mechanochemistry for the Barbier-type zinc-mediated coupling of allyl halides to aldehydes and ketones $(\mathrm{C}$, Scheme 1$)$.

\section{RESULTS \& DISCUSSION}

To begin, our investigations commenced with a model set of substrates, tolualdehyde (1) and ally bromide (2) with zinc flakes and a variety of stoichiometries, milling times, ball sizes/masses were investigated. We arrived at a molar ratio of regents of 1:1.5 (1:2) with two equivalents of zinc, and milling in a $10 \mathrm{~mL}$ jar for two hours with a single stainless steel ball of $9.25 \mathrm{~g}$ mass, which afforded the target allyl alcohol in $81 \%$ yield (Table 1, Entry 1). With these conditions in hand we looked to further improve the conversion of starting materials and explored the use of liquid assisted grinding agents (LAG) to refine the reaction outcome. Liquid assisted grinding, ${ }^{10}$ a technique more common in mechanochemical crystal engineering, ${ }^{11}$ is somewhat counterintuitive and requires the addition of a solvent-type species to reactions which would otherwise be solvent-free. 
Scheme 1. Context and Outline of Ball-milling ZincMediated Barbier-Type Reaction

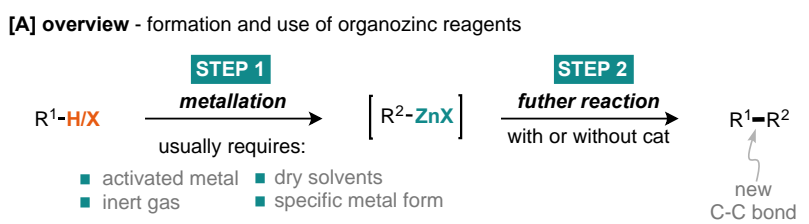

[B] previous mechanochemical work - Negishi reaction

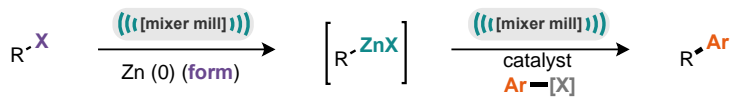

Reformatsky reaction

[20 examples, $\mathbf{s p}^{3}-\mathrm{sp}^{2}$ ]

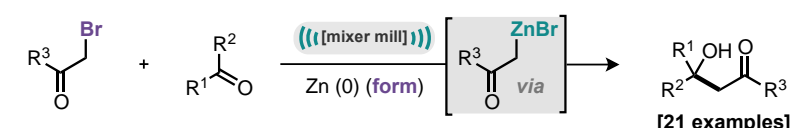

[C] this work - one-pot mechanochemical zinc-allylation reaction<smiles>C=CCBr</smiles>

$$
\mathrm{R}^{1} \stackrel{\mathrm{R}}{2} / \mathrm{H}_{\mathrm{O}}
$$

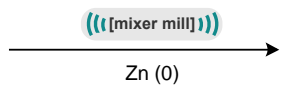

- applicable to all forms of zinc (0)

1. add reagents to milling jar

- solvent

2. screw jar closed (finger tight) 3. press play

Table 1. Optimization of Model Ball-milled Allylation Reaction

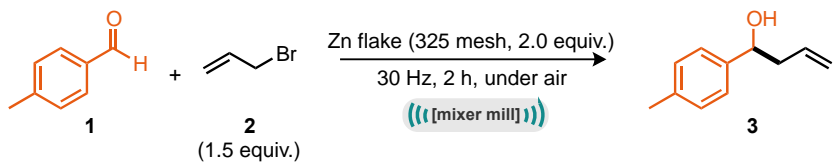

\begin{tabular}{ccc}
\hline Entry & variation from 'standard conditions' & Yield of $\mathbf{3}[\%]^{[\mathbf{a}]}$ \\
\hline 1 & none & 81 \\
2 & + DMA (1.5 equiv.) & 85 \\
3 & + DMF (1.5 equiv.) & 91 \\
4 & + DMSO (1.5 equiv.) & 99 \\
5 & + THF (1.5 equiv.) & 85 \\
6 & + DCM (1.5 equiv.) & 45 \\
7 & + Hexane (1.5 equiv.) & 51 \\
8 & + MeCN (1.5 equiv.) & 69 \\
9 & $\mathbf{2}(2.0$ equiv.) & 24 \\
\hline \multicolumn{4}{c}{} \\
\hline 10 & entries below this line contain DMSO (1.5 equiv.) \\
11 & 30 mins & 77 \\
12 & 1 h & 85 \\
\hline
\end{tabular}

${ }^{a}$ Isolated yields reported.

Typically, the amount of LAG used is assigned a value; $\eta$, which represents a liquid-to-reactant ratio; values between 0.1 and 1 describe reaction regimes within the $\mathrm{LAG}$ region as opposed to those that are neat $(\eta<0.1)$ and those that are slurries $(\eta>1)$ or solutions (typically when $\eta>10) .{ }^{10}$ The precise role of a LAG agent appears to be situation dependent, some have been found to facilitate access to different crystal polymorphic forms, ${ }^{11}$ whilst others have led to kinetic versus thermodynamic product outcomes depending on the dielectric constant of the LAG agent. ${ }^{12}$ Nonetheless, when screening a small range of LAG agents; representing a range of coordinating abilities and polarities, under the ball-milling Barbier-type reaction it was found that 1.5 equivalents of DMSO $(\eta=0.44)$ afforded the desired product in $99 \%$ yield (Table 1, Entry 4). Indeed, there appears to be a general trend that more coordinating liquid additives improve the reaction process, presuma- bly due to the breaking up aggregate organizinc species and ready access to exposed metal surface. Further investigation of the reaction time (Table 1, entires 10-12) with DMSO as LAG identified that two hours was optimal.

Next, our attention turned to exploring the substrate scope of the ball-mill enabled zinc-mediated Barbier-type reaction. Notably the reaction proceeds effectively against a range of aromatic aldehydes with halo substituents; iodo, bromo, chloro and fluoro were all tolerated under these conditions as well as a range of electron withdrawing groups and sterically encumbered mesitylaldehyde (A, Scheme 2). Notably 2formylpyridine and the electron rich 4(dimethylamino)benzaldehyde were not competent substrates in this reaction. A range of zinc sources was explored for the reaction of 4-fluorobenzaldehyde with allyl bromide and it was established that as well as zinc flake, zinc foil, zinc mesh, zinc wire and zinc shot are all effective under milling conditions, without any pre-treatment. The ball-milled process is not restricted to aromatic aldehydes with phenacetaldehyde (12; 47\%), cinnamaldehyde $(\mathbf{1 3} ; 84 \%)$, 2-phenylpropanal (14; 69\%, 3:1 anti:syn), hexanal $(\mathbf{1 5} ; 69 \%)$ and cyclohexanecarboxaldehyde $(\mathbf{1 6} ; 70 \%)$, all converting to the desired homoallylic alcohol products in moderate to good yields. Notably however crotylbromide and benzyl bromide were not effective substrates under these conditions. A range of 14 acetophenone substrates featuring electron rich, electron poor and sterically encumbered derivatives participated effectively in this process (B, Scheme 2$)$, so to did cyclohexenone $(22 ; 71 \%)$, cyclohexone $(27 ; 82 \%)$ and cyclooctone $(\mathbf{2 8} ; 94 \%)$. Imine electrophiles also participated in this reaction process to afford homoallylic amines in moderate to good yields (C, Scheme 2). 
Scheme 2. Scope of the ball-milling enable zinc-mediated Barbier reaction.
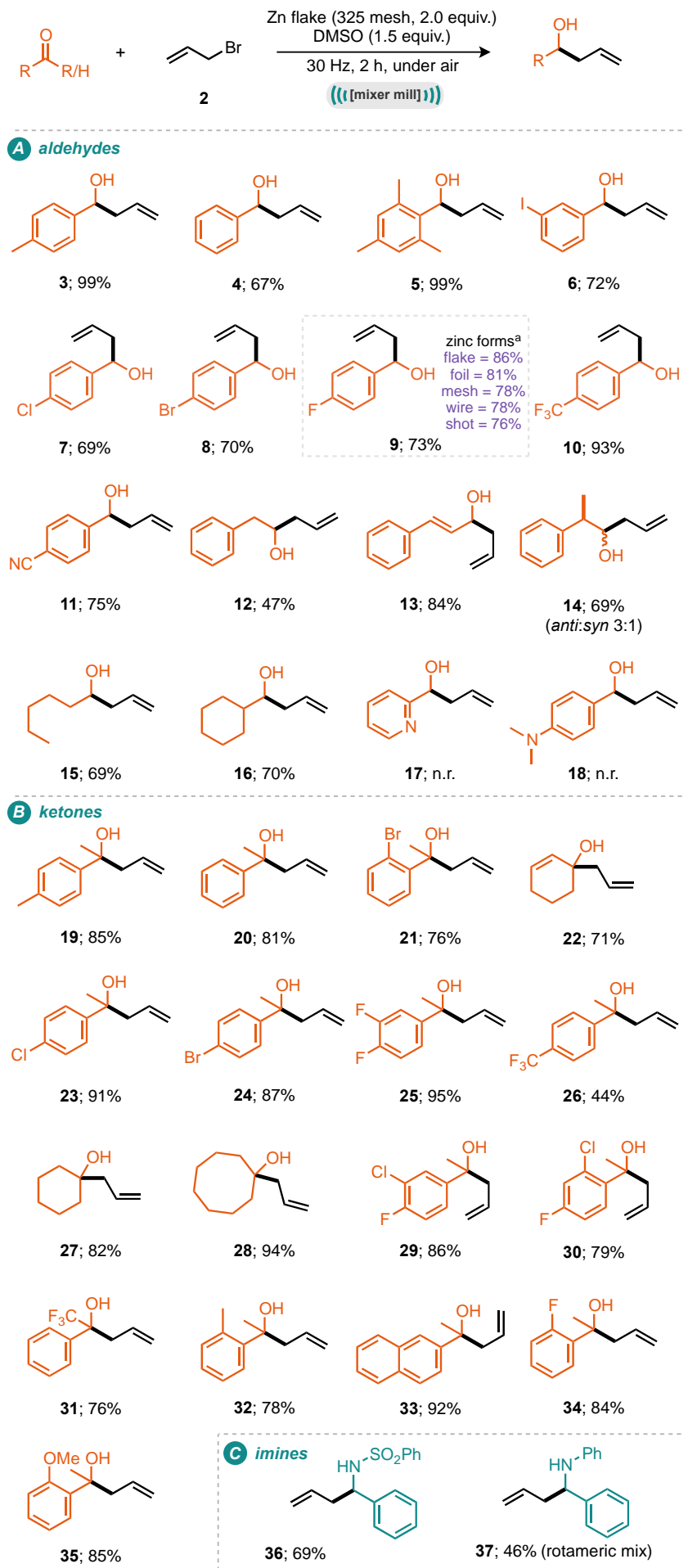

a) ${ }^{1} \mathrm{H}$ NMR yield with internal standard.

Alternative allylic and propargylic substrates were also investigated. It was found that allylchloride could effectively be used as pronucleophile in reaction with acetophenone to furnish the corresponding tertiary allylalcohol (20) in $85 \%$ yield (A, Scheme 3), although this reaction required six hours of milling to go to completion. Propargyl bromide underwent transformation to afford a 4:1 mixture of the homo-propargyl tertiary alcohol (40a) and corresponding allenyl derivative (40b) in $71 \%$ total yield.
Scheme 3. Further investigations

A other allylic/propargylic substrates<smiles>CC(=O)c1ccccc1</smiles><smiles>C=CC[18OH]</smiles>
$\begin{gathered}\text { Zn flake (325 mesh, } 2.0 \text { equiv.) } \\ \text { DMSO (1.2 equiv.) }\end{gathered}$
$\underset{\text { ((([mixer mill] })))}{30 \mathrm{~Hz}, \mathbf{6} \mathrm{h} \text {, under air }}$
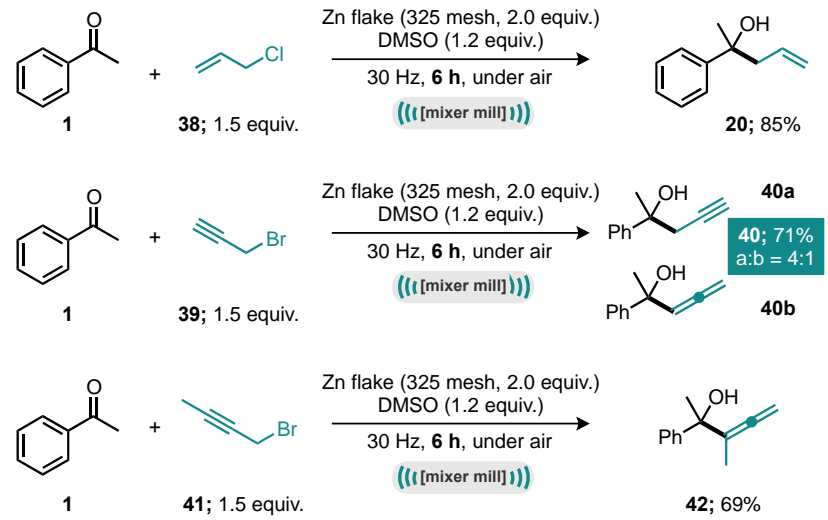

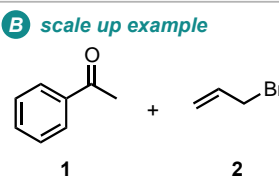
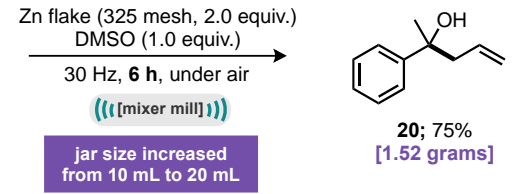

By replacing the terminal alkyne $\mathrm{C}-\mathrm{H}$ of propargyl bromide with a terminal methyl group through the use of 1-bromobutyne, $69 \%$ of the allenyl tertiary alcohol (42) could be isolate. The ball-milling process can also be scaled up, by simply changing to a larger jar $(25 \mathrm{~mL}$ rather than $10 \mathrm{~mL})$ and increasing the reaction time from two hours to six, we were able to isolate 1.52 grams of Barbier product $\mathbf{2 0}$.

To conclude, an operationally simple Barbier-type zincmediated mechanochemical protocol has been developed with good substrate scope and is applicable across a range of different zinc metal morphologies. The developed process, irrespective of the potentially complex behaviour of allyl zinc species, ${ }^{13}$ does not require dry solvents or inert atmospheres and can be straightforwardly scaled to deliver gram quantities of material. Furthermore in comparison to earlier work by Suzuki and co-workers using bismuth to mediate this process by ballmilling, ${ }^{3 j}$ the present method offers improved substrates scope and greatly reduced loading of metal, albeit at increased reaction time.

\section{EXPERIMENTAL SECTION}

General Information. Unless otherwise stated, all reagents were purchased from commercial sources and used without further purification. DMSO was purchased from Fluorochem (99\% purity). Different zinc forms were purchased from different companies as listed below: (1) Zinc granular (20-30 mesh, ACS reagent, $\geq 98.8 \%$; SigmaAldrich). (2) Zinc flake (-325 mesh, 99.9\%; Alfa Aesar). (3) Zinc foil (thickness $0.25 \mathrm{~mm}, 99.9 \%$ trace metals basis; Sigma-Aldrich). (4) Zinc shot (10 mm diameter x $2 \mathrm{~mm}$ thick, 99.99\%, Alfa Aesar). (5) Zinc wire (1.0 mm diameter, $99.95 \%$, Alfa Aesar).

Thin layer chromatography (TLC) was carried out using Merck TLC silica gel 60 sheet, and visualized with ultraviolet light or potassium permanganate stain. Flash column chromatography (FCC) was performed with Sigma Aldrich silica gel $40-60 \AA$ as the stationary phase and solvents employed were analytical grade. ${ }^{1} \mathrm{H}$ NMR spectra were recorded on a Bruker AVX500 (500 MHz) spectrometer at ambient temperature. ${ }^{13} \mathrm{C}$ NMR spectra were recorded on a Bruker AVX500 (125 MHz) spectrometer at ambient temperature. ${ }^{19} \mathrm{~F}$ NMR spectra were recorded on a Bruker AVX500 (471 MHz) spectrometer at ambient temperature. Melting points were measured on a Gallenkamp melting point apparatus and are reported corrected by 
linear calibration to benzophenone $\left(47-49^{\circ} \mathrm{C}\right)$ and benzoic acid $(121$ $\left.-123{ }^{\circ} \mathrm{C}\right)$.

High resolution mass spectroscopy (HRMS) data was obtained on a Thermo Scientific LTQ Orbitrap XL by the EPSRC UK National Mass Spectrometry Facility at Swansea University or on a Waters MALDI-TOF $m x$ in Cardiff University. Spectra were obtained using electron impact ionization (EI), chemical ionization (CI), positive electrospray (ES), pneumatically assisted electrospray (pNSI) or atmospheric solids analysis probe (ASAP+). Infrared spectra were recorded on a Shimadzu IR-Affinity-1S FTIR spectrometer.

The ball mill used was a Retsch MM 400 mixer mill. Unless otherwise stated, mechanochemical reactions were performed in $10 \mathrm{~mL}$ stainless steel jars from Retsch with a $12 \mathrm{~mm}$ diameter stainless steel ball $(\sim 9.25 \mathrm{~g})$. The longest time that this mill can be programmed to run for is 99 minutes. In order to run longer reaction times the mill was started, and then additional time added to the timer in order to ensure that the mill was running continuously for the desired reaction time.

General Procedure A for Reactions of Aldehydes and Allyl Bromide. To a Retsch $10 \mathrm{~mL}$ stainless steel milling jar was added the aldehyde (1.0 mmol, 1 equiv.), zinc (typically flake -325 mesh; 2.0 mmol, $131 \mathrm{mg}, 2$ equiv.), allyl bromide $(1.5 \mathrm{mmol}, 131 \mu \mathrm{L}, 1.5$ equiv.) and DMSO (1.5 mmol, $107 \mu 1,1.5$ equiv.) under air atmosphere. A $12 \mathrm{~mm}$ stainless steel ball was added and the mixture was milled at $30 \mathrm{~Hz}$ for 2 hours. After the reaction was finished, the resulting black paste was rinsed and transferred with ethyl acetate $(50 \mathrm{~mL})$ into a $100 \mathrm{~mL}$ conical flask, quenched with $1 \mathrm{M} \mathrm{HCl}(50 \mathrm{~mL})$ and stirred for 20 minutes. The organic layer was separated and dried over $\mathrm{MgSO}_{4}$, filtered and concentrated under reduced pressure. The crude material was then purified by silica gel flash chromatography.

General Procedure B for Reactions of Ketones and Allyl Bromide. To a Retsch $10 \mathrm{~mL}$ stainless steel milling jar was added the ketone (1.0 mmol, 1 equiv.), zinc (typically flake -325 mesh; $2.0 \mathrm{mmol}, 131$ mg, 2 equiv.), allyl bromide ( $1.5 \mathrm{mmol}, 131 \mu \mathrm{L} .1 .5$ equiv.) and DMSO (1.2 mmol, $85 \mu \mathrm{l}, 1.2$ equiv.) under air atmosphere. A $12 \mathrm{~mm}$ stainless steel ball was added and the mixture was milled at $30 \mathrm{~Hz}$ for 2 hours. After the reaction was finished, the resulting black paste was rinsed and transferred with ethyl acetate $(50 \mathrm{~mL})$ into a $100 \mathrm{~mL}$ conical flask, quenched with distilled water $(50 \mathrm{~mL})$ and stirred for at least 30 minutes. The mixture was then filtered through a pad of silica gel $(1.5 \mathrm{~cm})$ to remove insoluble materials. The silica gel was then flushed with ethyl acetate $(50 \mathrm{~mL})$, dried over $\mathrm{MgSO}_{4}$, filtered and concentrated under reduced pressure. The crude material was then purified by silica gel flash chromatography.

1-(p-tolyl)but-3-en-1-ol (3): Prepared according to General Procedure A. Purified by column chromatography (Ethyl Acetate/Hexane = $25: 75$ ) to give the product (yield: $99 \%, 160 \mathrm{mg}$ ) as a clear oil. Note: 3 is volatile under reduced pressure. ${ }^{1} \mathrm{H}$ NMR $\left(500 \mathrm{MHz}, \mathrm{CDCl}_{3}\right) \delta 7.22$ $(\mathrm{d}, J=8.1 \mathrm{~Hz}, 2 \mathrm{H}), 7.13(\mathrm{~d}, J=7.9 \mathrm{~Hz}, 2 \mathrm{H}), 5.81-5.73(\mathrm{~m}, 1 \mathrm{H}), 5.15-$ $5.08(\mathrm{~m}, 2 \mathrm{H}), 4.67(\mathrm{~m}, 1 \mathrm{H}), 2.49-2.45(\mathrm{~m}, 2 \mathrm{H}), 2.31(\mathrm{~s}, 3 \mathrm{H}), 1.96(\mathrm{~s}$, $1 \mathrm{H}) .{ }^{13} \mathrm{C}\{1 \mathrm{H}\} \mathrm{NMR}\left(126 \mathrm{MHz}, \mathrm{CDCl}_{3}\right) \delta 141.1,137.4,134.7,125.9$, $118.4,73.3,43.9,21.3$. Data is consistent with literature values. ${ }^{4 b, 14}$

1-phenylbut-3-en-1-ol (4): Prepared according to General Procedure A. Purified by column chromatography (Ethyl Acetate/Hexane = $20: 80$ ) to give the product (yield: $67 \%, 99 \mathrm{mg}$ ) as a clear oil. Note: 4 is volatile under reduced pressure. ${ }^{1} \mathrm{H}$ NMR $\left(500 \mathrm{MHz}, \mathrm{CDCl}_{3}\right) \delta$ 7.25-7.24 (m, 4H), 7.20-7.16 (m, 1H), 5.74-5.66 (ddt, $J=17.2,10.2$, $7.1 \mathrm{~Hz}, 1 \mathrm{H}), 5.07-5.02(\mathrm{~m} 2 \mathrm{H}), 4.61(\mathrm{dd}, J=7.4,5.6 \mathrm{~Hz}, 1 \mathrm{H}), 2.45-$ $2.36(\mathrm{~m}, 2 \mathrm{H}), 2.18$ (app. s, $1 \mathrm{H}) .{ }^{13} \mathrm{C}\{1 \mathrm{H}\}$ NMR $\left(126 \mathrm{MHz}, \mathrm{CDCl}_{3}\right) \delta$ 144.0, 134.6, 128.5, 127.6, 125.9, 118.4, 73.4, 43.9. Data is consistent with literature values. $4 \mathrm{~b}, 14$

1-mesitylbut-3-en-1-ol (5): Prepared according to General Procedure A. Purified by column chromatography (Ethyl Acetate/Hexane = $15: 85$ ) to give the product (yield: $99 \%, 188 \mathrm{mg}$ ) as a clear oil. ${ }^{1} \mathrm{H}$ NMR (500 MHz, $\left.\mathrm{CDCl}_{3}\right) \delta 6.85$ (app. s, 3H), 5.91-5.83 (m, 1H), 5.22$5.14(\mathrm{~m}, 3 \mathrm{H}), 2.77-2.70(\mathrm{~m}, 1 \mathrm{H}), 2.54-2.49(\mathrm{~m}, 1 \mathrm{H}), 2.44(\mathrm{~s}, 6 \mathrm{H}), 2.28$ $(\mathrm{s}, 3 \mathrm{H}), 1.97(\mathrm{~s}, 1 \mathrm{H}),{ }^{13} \mathrm{C}\{1 \mathrm{H}\}$ NMR $\left(126 \mathrm{MHz}, \mathrm{CDCl}_{3}\right) \delta$ 136.6, $136.1,135.4,130.2,117.8,70.8,40.4,20.9$. Data is consistent with literature values. ${ }^{14}$

1-(3-iodophenyl)but-3-en-1-ol (6): Prepared according to General Procedure A. Purified by column chromatography (Ethyl Acetate/Hexane $=15: 85$ ) to give the product (yield: $72 \%, 197 \mathrm{mg}$ ) as a clear oil. ${ }^{1} \mathrm{H}$ NMR $\left(500 \mathrm{MHz}, \mathrm{CDCl}_{3}\right) \delta 7.58(\mathrm{~s}, 1 \mathrm{H}), 7.48(\mathrm{~d}, J=7.8$ $\mathrm{Hz}, 1 \mathrm{H}), 7.16(\mathrm{~d}, J=7.7 \mathrm{~Hz}, 1 \mathrm{H}), 6.95(\mathrm{t}, J=7.8 \mathrm{~Hz}, 1 \mathrm{H}), 5.68-5.60$ $(\mathrm{m}, 1 \mathrm{H}), 5.03(\mathrm{~m}, 2 \mathrm{H}), 4.49$ (dd, $J=7.6,5.3 \mathrm{~Hz}, 1 \mathrm{H}), 2.53$ (broad s, $1 \mathrm{H}), 2.37-2.27(\mathrm{~m}, 2 \mathrm{H}) .{ }^{13} \mathrm{C}\{1 \mathrm{H}\} \mathrm{NMR}\left(126 \mathrm{MHz}, \mathrm{CDCl}_{3}\right) \delta 146.2$, 136.5, 134.8, 133.9, 130.1, 125.1, 118.7, 94.5, 72.4, 43.7. IR $\left(\mathrm{CH}_{2} \mathrm{Cl}_{2}\right.$ film): 1639, 1591, 1566, 1472, 1423, 1192, 1061, 995, 918, 781, 696 $\mathrm{cm}^{-1}$. HRMS (TOF-EI+) m/z: [M]+ calcd for $\mathrm{C}_{10} \mathrm{H}_{11} \mathrm{OI} 273.9855$; found 273.9846 .

1-(4-chlorophenyl)but-3-en-1-ol (7): Prepared according to General Procedure A. Purified by column chromatography (Ethyl Acetate $/$ Hexane $=15: 85 \rightarrow 20: 80$ ) to give the product (yield: $69 \%, 126$ $\mathrm{mg})$ as a clear oil. ${ }^{1} \mathrm{H}$ NMR $\left(500 \mathrm{MHz}, \mathrm{CDCl}_{3}\right) \delta$ 7.33-7.28 (m, 4H), 5.82-5.74 (m, 1H), 5.18-5.15 (m, 2H), 4.74-4.71 (m, 1H), 2.53-2.42 $(\mathrm{m}, 2 \mathrm{H}), 2.06(\mathrm{~s}, 1 \mathrm{H}) .{ }^{13} \mathrm{C}\{1 \mathrm{H}\}$ NMR $\left(126 \mathrm{MHz}, \mathrm{CDCl}_{3}\right) \delta 142.4$, $134.1,133.3,128.7,127.3,119.0,72.7,44.0$. Data is consistent with

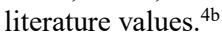

1-(4-bromophenyl)but-3-en-1-ol (8): Prepared according to General Procedure A. Purified by column chromatography (Ethyl Acetate $/$ Hexane $=10: 90 \rightarrow 20: 80$ ) to give the product (yield: $70 \%, 158$ $\mathrm{mg})$ as a clear oil. ${ }^{1} \mathrm{H}$ NMR $\left(500 \mathrm{MHz}, \mathrm{CDCl}_{3}\right) \delta 7.48(\mathrm{~d}, \mathrm{~J}=7.2 \mathrm{~Hz}$, $2 \mathrm{H}), 7.25(\mathrm{~m}, 2 \mathrm{H}), 5.82-5.74(\mathrm{~m}, 1 \mathrm{H}), 5.18-5.15(\mathrm{~m}, 2 \mathrm{H}), 4.71(\mathrm{~s}$, $1 \mathrm{H}), 2.53(\mathrm{~m}, 2 \mathrm{H}), 2.05(\mathrm{~s}, 1 \mathrm{H}) .{ }^{13} \mathrm{C}\{1 \mathrm{H}\}$ NMR $\left(126 \mathrm{MHz}, \mathrm{CDCl}_{3}\right) \delta$ 143.0, 134.1, 131.6, 127.7, 121.4, 119.1, 72.7, 44.0. Data is consistent with literature values. ${ }^{15}$

1-(4-fluorophenyl)but-3-en-1-ol (9): Prepared according to General Procedure A. Purified by column chromatography (Ethyl Acetate $/$ Hexane $=15: 85 \rightarrow 20: 80$ ) to give the product (yield: $73 \%, 121$ $\mathrm{mg}$ ) as a clear oil. Note: 9 is volatile under reduced pressure. ${ }^{1} \mathrm{H}$ NMR $\left(500 \mathrm{MHz}, \mathrm{CDCl}_{3}\right) \delta 7.33(\mathrm{dd}, J=8.2,5.7 \mathrm{~Hz}, 2 \mathrm{H}), 7.03(\mathrm{t}, J=8.6$ $\mathrm{Hz}, 2 \mathrm{H}), 5.79(\mathrm{~m}, 1 \mathrm{H}), 5.18-5.14(\mathrm{~m}, 2 \mathrm{H}), 4.73(\mathrm{dd} J=6.8,6.0 \mathrm{~Hz}$, $1 \mathrm{H}), 2.54-2.44(\mathrm{~m}, 2 \mathrm{H}), 2.05(\mathrm{~s}, 1 \mathrm{H}) .{ }^{13} \mathrm{C}\{1 \mathrm{H}\} \mathrm{NMR}(126 \mathrm{MHz}$, $\left.\mathrm{CDCl}_{3}\right) \delta 162.3(\mathrm{~d}, J=246 \mathrm{~Hz}), 139.7(\mathrm{~d}, J=2.5 \mathrm{~Hz}), 134.3,127.6$, $118.9,115.6(\mathrm{~d}, J=21.4 \mathrm{~Hz}), 72.8,44.1 .{ }^{19} \mathrm{~F} \mathrm{NMR}\left(376 \mathrm{MHz}, \mathrm{CDCl}_{3}\right)$ $\delta$-115.2. Data is consistent with literature values. ${ }^{15}$

1-(4-(trifluoromethyl)phenyl)but-3-en-1-ol (10): Prepared according to General Procedure A. Purified by column chromatography (Ethyl Acetate/Hexane $=10: 90 \rightarrow 20: 80$ ) to give the product (yield: $91 \%, 201$ $\mathrm{mg})$ as a clear oil. ${ }^{1} \mathrm{H}$ NMR $\left(500 \mathrm{MHz}, \mathrm{CDCl}_{3}\right) \delta 7.61(\mathrm{~d}, J=8.1 \mathrm{~Hz}$, $2 \mathrm{H}), 7.48(\mathrm{~d}, J=8.0 \mathrm{~Hz}, 2 \mathrm{H}), 5.84-5.75(\mathrm{~m}, 1 \mathrm{H}), 5.18(\mathrm{~d}, J=12.3 \mathrm{~Hz}$, $2 \mathrm{H}), 4.82-4.80(\mathrm{~m}, 1 \mathrm{H}), 2.58-2.53(\mathrm{~m}, 1 \mathrm{H}), 2.50-2.44(\mathrm{~m}, 1 \mathrm{H}), 2.13$ $(\mathrm{s}, 1 \mathrm{H}) \cdot{ }^{13} \mathrm{C}\{1 \mathrm{H}\}$ NMR $\left(126 \mathrm{MHz}, \mathrm{CDCl}_{3}\right) \delta 147.9,133.8,129.9(\mathrm{q}$, $J=33 \mathrm{~Hz}), 126.2,125.5,123.2,119.4,72.7,44.1 .{ }^{19} \mathrm{~F}$ NMR $(376$ $\left.\mathrm{MHz}, \mathrm{CDCl}_{3}\right) \delta-62.5$. Data is consistent with literature values. ${ }^{14}$

4-(1-hydroxybut-3-en-1-yl)benzonitrile (11): Prepared according to General Procedure A. Purified by column chromatography (Ethyl Acetate $/$ Hexane $=15: 85$ ) to give the product (yield: $75 \%, 130 \mathrm{mg}$ ) as a clear oil. ${ }^{1} \mathrm{H}$ NMR $\left(500 \mathrm{MHz}, \mathrm{CDCl}_{3}\right) \delta 7.61(\mathrm{~m}, 2 \mathrm{H}), 7.46(\mathrm{~m}, 2 \mathrm{H})$, 5.80-5.71 (m, 1H), 5.16-5.12 (m, 2H), 4.80-4.76 (m, 1H), 2.54-2.48 $(\mathrm{m}, 2 \mathrm{H}), 2.46-2.39(\mathrm{~m}, 1 \mathrm{H}) .{ }^{13} \mathrm{C}\{1 \mathrm{H}\}$ NMR $\left(126 \mathrm{MHz}, \mathrm{CDCl}_{3}\right) \delta$ $149.3,133.5,132.3,126.6,119.4,119.0,111.1,72.5,43.9$. Data is consistent with literature values but the NMR data indicates presence of minor impurities. ${ }^{4 \mathrm{~b}}$

1-phenylpent-4-en-2-ol (12): Prepared according to General Procedure A. Purified by column chromatography (Ethyl Acetate/Hexane = $15: 85$ ) to give the product (yield: $47 \%, 76 \mathrm{mg}$ ) as a clear oil. Note: 12 is volatile under reduced pressure. ${ }^{1} \mathrm{H}$ NMR $\left(500 \mathrm{MHz}, \mathrm{CDCl}_{3}\right) \delta$ 7.25-7.21 (m, 2H), 7.17-7.13 (m, 3H), 5.84-5.73 (m, 1H), 5.10-5.05 $(\mathrm{m}, 2 \mathrm{H}), 3.83-3.77(\mathrm{~m}, 1 \mathrm{H})$ or $3.80(\mathrm{tt}, 7.7,4.8 \mathrm{~Hz}, 1 \mathrm{H}), 2.74(\mathrm{dd}, J=$ $13.6,4.9 \mathrm{~Hz}, 1 \mathrm{H}), 2.64(\mathrm{dd}, J=13.6,7.9 \mathrm{~Hz}, 1 \mathrm{H}), 2.29-2.18(\mathrm{~m}, 1 \mathrm{H})$, 2.16-2.08 (m, 1H), $1.66(\mathrm{~s}, 1 \mathrm{H}) .{ }^{13} \mathrm{C}\{1 \mathrm{H}\}$ NMR $\left(126 \mathrm{MHz}, \mathrm{CDCl}_{3}\right) \delta$ $138.5,134.8,129.5,128.6,126.6,118.2,71.8,43.4,41.3$. Data is consistent with literature values. ${ }^{16}$

(E)-1-phenylhexa-1,5-dien-3-ol (13): Prepared according to General Procedure A. Purified by column chromatography (Ethyl Acetate/Hexane $=15: 85$ ) to give the product (yield: $84 \%, 146 \mathrm{mg}$ ) as a clear oil. ${ }^{1} \mathrm{H}$ NMR $\left(500 \mathrm{MHz}, \mathrm{CDCl}_{3}\right) \delta 7.28(\mathrm{~m}, 2 \mathrm{H}), 7.22-7.19(\mathrm{~m}$, $2 \mathrm{H}), 7.15-7.12(\mathrm{~m}, 1 \mathrm{H}), 6.50(\mathrm{~d}, J=15.6 \mathrm{~Hz}, 1 \mathrm{H}), 6.14(\mathrm{dd}, J=15.9$, $6.4 \mathrm{~Hz}, 1 \mathrm{H}), 5.76$ (ddt, 17.2, 10.2, 7.1 Hz, 1H), 5.10-5.04 (m, 2H), $4.25(\mathrm{q}, J=5.9 \mathrm{~Hz}, 1 \mathrm{H}), 2.31-2.25(\mathrm{~m}, 2 \mathrm{H}), 2.05(\mathrm{~s}, 1 \mathrm{H}) .{ }^{13} \mathrm{C}\{1 \mathrm{H}\}$ NMR $\left(126 \mathrm{MHz}, \mathrm{CDCl}_{3}\right) \delta 136.7,134.2,131.7,130.4,128.6,127.7$, $126.6,118.4,71.8,42.1$. Data is consistent with literature values. ${ }^{14}$ 
2-phenylhex-5-en-3-ol (14): Prepared according to General Procedure A. Purified by column chromatography (Ethyl Acetate/Hexane = 20:80) to give the product (yield: $69 \%, 122 \mathrm{mg}$ ) as a clear oil. Note: data was reported here as a mixture of syn and anti diastereomers. ${ }^{1} \mathrm{H}$ NMR (500 MHz, $\left.\mathrm{CDCl}_{3}\right) \delta 7.25-7.11(\mathrm{~m}, 5 \mathrm{H}$, syn and anti), 5.84-5.77 (m, 1H, syn), 5.76-5.67 (m, 1H, anti), 5.06-4.98 (m, 2H, syn and anti), 3.65-3.60 $(\mathrm{m}, 1 \mathrm{H}$, syn and anti), 2.75-2.64 $(\mathrm{m}, 1 \mathrm{H}$, syn and anti), 2.31-2.27 (m, 1H, syn), 2.13-2.07 (m, 1H, anti), 2.06-1.97 (m, 1H, syn), 1.96-1.91 (m, 1H, anti), $1.69(\mathrm{~s}, 1 \mathrm{H}$, anti $), 1.60(\mathrm{~s}, 1 \mathrm{H}$, ant $), 1.26$ (d, $J=7.0 \mathrm{~Hz}, 3 \mathrm{H}$, anti), $1.21\left(\mathrm{~d}, J=7.1 \mathrm{~Hz}\right.$, anti). ${ }^{13} \mathrm{C}\{1 \mathrm{H}\} \mathrm{NMR}$ $\left(126 \mathrm{MHz}, \mathrm{CDCl}_{3}\right) \delta 144.5,143.4,135.2,135.1,128.6,128.6,128.3$, $127.9,126.7,126.5,118.0,117.8,75.1,75.1,45.5,45.5,39.6,39.0$, 17.8. 16.5. Data is consistent with literature values. ${ }^{17,18}$

Non-1-en-4-ol (15): Prepared according to General Procedure A. Purified by column chromatography (Ethyl Acetate/Hexane $=10: 90)$ to give the product (yield: $69 \%, 98 \mathrm{mg}$ ) as a clear oil. Note: 15 is volatile under reduced pressure. ${ }^{1} \mathrm{H}$ NMR $\left(500 \mathrm{MHz}, \mathrm{CDCl}_{3}\right) \delta 5.86-$ $5.78(\mathrm{~m}, 1 \mathrm{H}), 5.13-5.10(\mathrm{~m}, 2 \mathrm{H}), 3.65-3.61(\mathrm{~m}, 1 \mathrm{H}), 2.31-2.26(\mathrm{~m}$ $1 \mathrm{H}), 2.16-2.10(\mathrm{~m}, 1 \mathrm{H}), 1.71$ (broad s, $1 \mathrm{H}), 1.45-1.25(\mathrm{~m}, 8 \mathrm{H}), 0.88$ $(\mathrm{t}, J=6.9 \mathrm{~Hz}, 3 \mathrm{H}) \cdot{ }^{13} \mathrm{C}\{1 \mathrm{H}\} \mathrm{NMR}\left(126 \mathrm{MHz}, \mathrm{CDCl}_{3}\right) \delta 135.1,118.1$, $70.8,42.1,36.9,32.0,25.5,22.8,14.2$. Data is consistent with literature values but the NMR data indicates presence of minor impurities. $^{14}$

1-cyclohexylbut-3-en-1-ol (16): Prepared according to General Procedure A. Purified by column chromatography (Ethyl Acetate/Hexane $=20: 80$ ) to give the product (yield: $70 \%, 108 \mathrm{mg}$ ) as a clear oil. Note: 16 is volatile under reduced pressure. ${ }^{1} \mathrm{H}$ NMR $\left(500 \mathrm{MHz}, \mathrm{CDCl}_{3}\right) \delta$ 5.87-5.77 (m, 1H), 5.14-2.09 (m, 2H), 3.39-3.35 (m, 1H), 2.34-2.28 (m, 1H), 2.15-2.07 (m, 1H), 1.86-1.64 (m, 6H), 1.35-0.98 (m, 6H). ${ }^{13} \mathrm{C}\{1 \mathrm{H}\}$ NMR $\left(126 \mathrm{MHz}, \mathrm{CDCl}_{3}\right) \delta 135.6,117.9,74.84,43.2,38.9$, $29.2,28.2,26.6,26.4,26.2$. Data is consistent with literature values.

2-(p-tolyl)pent-4-en-2-ol (19): Prepared according to General Procedure B. Purified by column chromatography (Ethyl Acetate/Hexane $=20: 80$ ) to give the product (yield: $85 \%, 150 \mathrm{mg}$ ) as a clear oil. Note: 19 is volatile under reduced pressure. ${ }^{1} \mathrm{H}$ NMR $\left(500 \mathrm{MHz}, \mathrm{CDCl}_{3}\right) \delta$ $7.34(\mathrm{~d}, \mathrm{~J}=8.1 \mathrm{~Hz}, 2 \mathrm{H}), 7.17(\mathrm{~d}, \mathrm{~J}=8.0 \mathrm{~Hz}, 2 \mathrm{H}), 5.69-5.60(\mathrm{~m}, 1 \mathrm{H})$, 5.17-5.11 (m, 2H), 2.71-2.67 (m, 1H), 2.53-2.48 (m, 1H), $2.35(\mathrm{~s}$, $3 \mathrm{H}), 2.08(\mathrm{~s}, 1 \mathrm{H}), 1.54(\mathrm{~s}, 3 \mathrm{H}),{ }^{13} \mathrm{C}\{1 \mathrm{H}\}$ NMR $\left(126 \mathrm{MHz}, \mathrm{CDCl}_{3}\right) \delta$ $144.8,136.3,133.9,129.0,124.8,119.4,73.6,48.6,30.1,21.1$. Data is consistent with literature values. ${ }^{19}$

2-phenylpent-4-en-2-ol (20): Prepared according to General Procedure B. Purified by column chromatography (Ethyl Acetate/Hexane $=$ $20: 80$ ) to give the product (yield: $81 \%, 131 \mathrm{mg}$ ) as a clear oil. Note: 20 is volatile under reduced pressure. $7 \%$ unreacted acetophenone was present in the purified NMR. ${ }^{1} \mathrm{H}$ NMR $\left(500 \mathrm{MHz}, \mathrm{CDCl}_{3}\right) \delta 7.50-$ $7.47(\mathrm{~m}, 2 \mathrm{H}), 7.41-7.37(\mathrm{~m}, 2 \mathrm{H}), 7.30-7.26(\mathrm{~m}, 1 \mathrm{H})$, 5.71-5.63 $(\mathrm{m}$, $1 \mathrm{H}), 5.20-5.14(\mathrm{~m}, 2 \mathrm{H}), 2.76-2.70(\mathrm{~m}, 1 \mathrm{H}), 2.58-2.52(\mathrm{~m}, 1 \mathrm{H}), 2.26$ (broad s, 1H), $1.59(\mathrm{~s}, 3 \mathrm{H}),{ }^{13} \mathrm{C}\{1 \mathrm{H}\}$ NMR $\left(126 \mathrm{MHz}, \mathrm{CDCl}_{3}\right) \delta$ $147.1,133.8,128.2,126.7,124.9,119.4,73.7,48.6,29.9$. Data is consistent with literature values. ${ }^{19}$

2-(2-bromophenyl)pent-4-en-2-ol (21): Prepared according to General Procedure B. Purified by column chromatography (Ethyl Acetate $/$ Hexane $=15: 85$ ) to give the product (yield: $76 \%, 182 \mathrm{mg}$ ) as a clear oil. ${ }^{1} \mathrm{H}$ NMR $\left(500 \mathrm{MHz}, \mathrm{CDCl}_{3}\right) \delta 7.71(\mathrm{dd}, J=8.0,1.7 \mathrm{~Hz}$, $1 \mathrm{H}), 7.58$ (dd, $J=7.9,1.3 \mathrm{~Hz}, 1 \mathrm{H}), 7.30$ (dt, $J=7.4,1.3 \mathrm{~Hz}, 1 \mathrm{H}), 7.09$ $(\mathrm{dt}, J=7.4,1.7 \mathrm{~Hz}, 1 \mathrm{H}), 5.57$ (dddd, $J=17.1,10.1,8.4,6.4 \mathrm{~Hz}, 1 \mathrm{H}$ ), $5.19-5.07(\mathrm{~m}, 2 \mathrm{H}), 3.33-3.23(\mathrm{~m}, 1 \mathrm{H}), 2.70(\mathrm{~s}, 1 \mathrm{H}), 2.69-2.63$ $(\mathrm{m}, 1 \mathrm{H}), 1.73(\mathrm{~s}, 3 \mathrm{H}) .{ }^{13} \mathrm{C}\{1 \mathrm{H}\}$ NMR $\left(126 \mathrm{MHz}, \mathrm{CDCl}_{3}\right) \delta 145.1$, 135.1, 133.7, 128.6, 128.3, 127.5, 120.0, 119.4, 74.7, 45.1, 27.4. Data is consistent with literature values. ${ }^{20}$

1-allylcyclohex-2-en-1-ol (22): Prepared according to General Procedure B. Purified by column chromatography (Ethyl Acetate/Hexane $=15: 85$ ) to give the product (yield: $71 \%, 98 \mathrm{mg}$ ) as a clear oil. Note: 22 is volatile under reduced pressure. ${ }^{1} \mathrm{H}$ NMR $\left(500 \mathrm{MHz}, \mathrm{CDCl}_{3}\right) \delta$ 5.93-5.80 (m, 2H), 5.65-5.61 (m, 1H), 5.16-5.13 (m, 2H), $2.31(\mathrm{~m}$, $2 \mathrm{H}), 2.11-1.86(\mathrm{~m}, 2 \mathrm{H}), 1.76-1.63(\mathrm{~m}, 5 \mathrm{H}),{ }^{13} \mathrm{C}\{1 \mathrm{H}\}$ NMR $(126$ $\left.\mathrm{MHz}, \mathrm{CDCl}_{3}\right) \delta 133.8,132.3,130.4,118.8,69.3,46.9,35.7,35.7$, $19.9,25.3,19.1$. Data is consistent with literature values. ${ }^{21}$

2-(4-chlorophenyl)pent-4-en-2-ol (23) Prepared according to General Procedure B. Purified by column chromatography (Ethyl Acetate/Hexane $=20: 80$ ) to give the product (yield: $91 \%, 178 \mathrm{mg}$ ) as a clear oil. ${ }^{1} \mathrm{H}$ NMR $\left(500 \mathrm{MHz}, \mathrm{CDCl}_{3}\right) \delta$ 7.38-7.36 (m, 2H), 7.31-7.29 (m, $2 \mathrm{H}$ ), 5.60 (dddd, $J=20.2,9.5,8.2,6.5 \mathrm{~Hz}, 1 \mathrm{H}), 5.13$ (dd, $J=$ 14.0, $1.3 \mathrm{~Hz}, 2 \mathrm{H}$ ), 2.64 (dd, $J=13.8,6.5 \mathrm{~Hz}, 1 \mathrm{H}$ ), 2.48 (dd, $J=13.8$, $8.3 \mathrm{~Hz}, 1 \mathrm{H}), 2.13(\mathrm{~s}, 1 \mathrm{H}), 1.52(\mathrm{~s}, 3 \mathrm{H}) .{ }^{13} \mathrm{C}\{1 \mathrm{H}\}$ NMR $(126 \mathrm{MHz}$, $\left.\mathrm{CDCl}_{3}\right) \delta 146.3,133.3,132.5,128.4,126.5,120.0,73.5,48.5,30.0$. Data is consistent with literature values. ${ }^{19}$

2-(4-bromophenyl)pent-4-en-2-ol (24): Prepared according to General Procedure B. Purified by column chromatography (Ethyl Acetate/Hexane $=20: 80$ ) to give the product (yield: $87 \%, 209 \mathrm{mg}$ ) as a clear oil. Note: trace 4'-Bromoacetophenone was observed in the purified NMR. ${ }^{1} \mathrm{H}$ NMR $\left(500 \mathrm{MHz}, \mathrm{CDCl}_{3}\right) \delta$ 7.44-7.42 (d, $J=8.8$ $\mathrm{Hz}, 2 \mathrm{H}), 7.33-7.31$ (d, $J=8.8 \mathrm{~Hz}, 2 \mathrm{H}), 5.61-5.51(\mathrm{~m}, 1 \mathrm{H}), 5.11(\mathrm{~d}, J=$ $12.4 \mathrm{~Hz}, 2 \mathrm{H}), 2.63(\mathrm{dd}, J=13.7,6.5 \mathrm{~Hz}, 1 \mathrm{H}), 2.49(\mathrm{dd}, J=13.7,8.2$ $\mathrm{Hz}, 1 \mathrm{H}), 2.11(\mathrm{~s}, 1 \mathrm{H}), 1.52(\mathrm{~s}, 3 \mathrm{H}) \cdot{ }^{13} \mathrm{C}\{1 \mathrm{H}\} \mathrm{NMR}\left(126 \mathrm{MHz}, \mathrm{CDCl}_{3}\right)$ $\delta 146.8,133.3,131.3,126.9,120.7,120.0,73.5,48.4,30.0$. Data is consistent with literature values. ${ }^{20}$

2-(3,4-difluorophenyl)pent-4-en-2-ol (25): Prepared according to General Procedure B. Purified by column chromatography (Ethyl Acetate/Hexane $=15: 85$ ) to give the product (yield: $95 \%, 188 \mathrm{mg}$ ) as a clear oil. ${ }^{1} \mathrm{H}$ NMR $\left(500 \mathrm{MHz}, \mathrm{CDCl}_{3}\right) \delta$ 7.31-7.27 (m, $\left.1 \mathrm{H}\right), 7.15-$ $7.09(\mathrm{~m}, 2 \mathrm{H}), 5.62(\mathrm{~m}, 1 \mathrm{H}), 5.16-5.13(\mathrm{~m}, 2 \mathrm{H}), 2.63(\mathrm{dd}, \mathrm{J}=13.8,6.6$ $\mathrm{Hz}, 1 \mathrm{H}), 2.49(\mathrm{dd}, \mathrm{J}=13.8,8.2 \mathrm{~Hz}, 1 \mathrm{H}), 2.22($ broad s, $1 \mathrm{H}), 1.53(\mathrm{~s}$, $3 \mathrm{H}) .{ }^{13} \mathrm{C}\{1 \mathrm{H}\}$ NMR $\left(126 \mathrm{MHz}, \mathrm{CDCl}_{3}\right) \delta 150.1(\mathrm{dd}, J=16.4,247.0$ $\mathrm{Hz}), 149.1(\mathrm{dd}, J=13.9,248.2 \mathrm{~Hz}), 145.0$ (t, $J=44.0 \mathrm{~Hz}), 133.1$, $120.9(\mathrm{q}, J=33.6 \mathrm{~Hz}), 120.1,116.9(\mathrm{~d}, J=17.6 \mathrm{~Hz}), 114.4(\mathrm{~d}, J=$ $17.6 \mathrm{~Hz}), 73.3$, 48.5, 29.9. IR $\left(\mathrm{CH}_{2} \mathrm{Cl}_{2}\right.$ film): 2984, 1609, 1514, 1418 , 1379, 1277, 1153, 1117, 947. 922, 818, $775 \mathrm{~cm}^{-1}$. HRMS (TOF-EI+) $\mathrm{m} / \mathrm{z}$ : [M- $\left.\mathrm{H}_{2} \mathrm{O}\right]+$ calcd for $\mathrm{C}_{11} \mathrm{H}_{10} \mathrm{~F}_{2}$ 180.0751; found 180.0747 .

2-(4-(trifluoromethyl)phenyl)pent-4-en-2-ol (26): Prepared according to General Procedure B. Purified by column chromatography (Ethyl Acetate/Hexane $=15: 85$ ) to give the product (yield: 44\%, 101 $\mathrm{mg})$ as a clear oil. ${ }^{1} \mathrm{H}$ NMR $\left(500 \mathrm{MHz}, \mathrm{CDCl}_{3}\right) \delta 7.61-7.55(\mathrm{~m}, 4 \mathrm{H})$, 5.64-5.55 (m, 1H), 5.17-5.13 (m, 2H), $2.68(\mathrm{dd}, J=13.8,6.4 \mathrm{~Hz}, 1 \mathrm{H})$, $2.52(\mathrm{dd}, J=13.8,8.3 \mathrm{~Hz}, 1 \mathrm{H}), 2.24(\mathrm{~s}, 1 \mathrm{H}), 1.56(\mathrm{~s}, 3 \mathrm{H}) .{ }^{13} \mathrm{C} \mathrm{NMR}$ $\left(126 \mathrm{MHz}, \mathrm{CDCl}_{3}\right) \delta 151.8(\mathrm{~d}, J=1.0 \mathrm{~Hz}), 133.1,129.0(\mathrm{q}, J=32.4$ $\mathrm{Hz}), 125.4,125.3(\mathrm{q}, J=3.8 \mathrm{~Hz}), 124.4(\mathrm{q}, J=271.8 \mathrm{~Hz}), 120.2,73.7$, 48.4, 30.0. ${ }^{13} \mathrm{C}\{1 \mathrm{H}\}$ NMR $\left(126 \mathrm{MHz}, \mathrm{CDCl}_{3}\right) \delta 147.9,133.8,129.9$ $(\mathrm{q}, J=32.4 \mathrm{~Hz}), 126.2,125.5(\mathrm{q}, J=3.8 \mathrm{~Hz}), 124.3(\mathrm{q}, J=271.9 \mathrm{~Hz})$, 119.4, 72.7, 44.1. IR ( $\mathrm{CH}_{2} \mathrm{Cl}_{2}$ film): 1379, 1327, 1165, 1125, 1070, $1015,955,939,843 \mathrm{~cm}^{-1}$. HRMS (TOF-EI+) m/z: [M- $\left.\mathrm{H}_{2} \mathrm{O}\right]+$ calcd for $\mathrm{C}_{12} \mathrm{H}_{9} \mathrm{~F}_{3} 212.0813$; found 212.0805 .

1-allylcyclohexan-1-ol (27): Prepared according to General Procedure B. Purified by column chromatography (Ethyl Acetate/Hexane = $15: 85$ ) to give the product (yield: $82 \%, 115 \mathrm{mg}$ ) as a clear oil. ${ }^{1} \mathrm{H}$ NMR $\left(500 \mathrm{MHz}, \mathrm{CDCl}_{3}\right) \delta 5.89(\mathrm{ddt}, J=17.7,10.2,7.5 \mathrm{~Hz}), 5.16-$ $5.09(\mathrm{~m}, 2 \mathrm{H}), 2.22(\mathrm{~d}, J=7.5 \mathrm{~Hz}, 2 \mathrm{H}), 1.64-1.40(\mathrm{~m}, 13 \mathrm{H}), 1.30-1.25$ $(\mathrm{m}, 2 \mathrm{H}) .{ }^{13} \mathrm{C}\{1 \mathrm{H}\}$ NMR $\left(126 \mathrm{MHz}, \mathrm{CDCl}_{3}\right) \delta 133.9,118.8,71.1$, $46.8,37.5,25.9,22.3$. Data is consistent with literature values. ${ }^{19}$

1-allylcyclooctan-1-ol (28): Prepared according to General Procedure B. Purified by column chromatography (Ethyl Acetate/Hexane $=$ 20:80) to give the product (yield: $94 \%, 158 \mathrm{mg}$ ) as a clear oil. ${ }^{1} \mathrm{H}$ NMR $\left(500 \mathrm{MHz}, \mathrm{CDCl}_{3}\right) \delta 5.83$ (ddt, 17.7, 10.2, 7.5 Hz, 1H), 5.09$5.02(\mathrm{~m}, 2 \mathrm{H}), 2.15(\mathrm{~d}, J=7.5 \mathrm{~Hz}), 1.59-1.35(\mathrm{~m}, 19 \mathrm{H}) .{ }^{13} \mathrm{C}\{1 \mathrm{H}\}$ NMR $\left(126 \mathrm{MHz}, \mathrm{CDCl}_{3}\right) \delta 134.2,118.7,74.5,46.1,36.3,28.4 .25 .1$, 22.3. Data is consistent with literature values.

2-(3-chloro-4-fluorophenyl)pent-4-en-2-ol (29): Prepared according to General Procedure B. Purified by column chromatography (Ethyl Acetate/Hexane $=20: 80$ ) to give the product (yield: $86 \%, 184 \mathrm{mg}$ ) as a clear oil. Note: trace 3'-Chloro-4'-fluoroacetophenone was observed in the purified NMR. ${ }^{1} \mathrm{H}$ NMR $\left(500 \mathrm{MHz}, \mathrm{CDCl}_{3}\right) \delta 7.52-7.50(\mathrm{~m}$, $1 \mathrm{H}), 7.31-7.27(\mathrm{~m}, 1 \mathrm{H}), 7.10(\mathrm{t}, J=8.7 \mathrm{~Hz}, 1 \mathrm{H}), 5.62(\mathrm{~m}, 1 \mathrm{H}), 5.17-$ $5.13(\mathrm{~m}, 2 \mathrm{H}), 2.63(\mathrm{dd}, J=13.8,6.6 \mathrm{~Hz}, 1 \mathrm{H}), 2.49(\mathrm{dd}, J=13.8,8.1$ $\mathrm{Hz}, 1 \mathrm{H}), 2.22(\mathrm{~s}, 1 \mathrm{H}), 1.53(\mathrm{~s}, 3 \mathrm{H}) \cdot{ }^{13} \mathrm{C}\{1 \mathrm{H}\} \mathrm{NMR}\left(126 \mathrm{MHz}, \mathrm{CDCl}_{3}\right)$ $\delta 156.8(\mathrm{~d}, J=248.2 \mathrm{~Hz}), 144.8,132.9,127.4,124.8,120.1,116.2$, 116.2, 73.2, 48.4, 29.9. IR ( $\mathrm{CH}_{2} \mathrm{Cl}_{2}$ film): 1684, 1639, 15.91, 1497, $1391,1263,1244,1076,1057,920,880,820,729,714 \mathrm{~cm}^{-1}$ HRMS (TOF-ES+) m/z: [M- $\left.\mathrm{H}_{2} \mathrm{O}+\mathrm{H}\right]+$ calcd for $\mathrm{C}_{11} \mathrm{H}_{11} \mathrm{ClF}$ 197.0533; found 197.0540

2-(2-chloro-4-fluorophenyl)pent-4-en-2-ol (30): Prepared according to General Procedure B. Purified by column chromatography (Ethyl Acetate $/$ Hexane $=20: 80$ ) to give the product (yield: $79 \%, 169 \mathrm{mg}$ ) as a clear oil. ${ }^{1} \mathrm{H}$ NMR $\left(500 \mathrm{MHz}, \mathrm{CDCl}_{3}\right) \delta 7.69(\mathrm{dd}, J=8.9,6.4 \mathrm{~Hz}$, 
$1 \mathrm{H}), 7.11$ (dd, $J=8.4,2.7 \mathrm{~Hz}, 1 \mathrm{H}), 6.97$ (ddd, $J=8.9,7.7,2.7 \mathrm{~Hz}$, $1 \mathrm{H}), 5.59-5.48(\mathrm{~m}, 1 \mathrm{H}), 5.18-5.06(\mathrm{~m}, 2 \mathrm{H}), 3.18(\mathrm{dd}, J=14.0,6.4$ $\mathrm{Hz}, 1 \mathrm{H}), 2.61$ (dd, $J=14.0,8.4 \mathrm{~Hz}, 1 \mathrm{H}), 2.46(\mathrm{~s}, 1 \mathrm{H}), 1.68$ (s, 3H). ${ }^{13} \mathrm{C}\{1 \mathrm{H}\}$ NMR $\left(126 \mathrm{MHz}, \mathrm{CDCl}_{3}\right) \delta 161.4(\mathrm{~d}, J=312.5 \mathrm{~Hz}), 139.8$ $(\mathrm{d}, J=5.0 \mathrm{~Hz}), 133.5,131.3(\mathrm{~d}, J=12.6 \mathrm{~Hz}), 129.4(\mathrm{~d}, J=10.1 \mathrm{~Hz})$, $119.8,118.5(\mathrm{~d}, J=31.5 \mathrm{~Hz}), 113.8(\mathrm{~d}, J=25.2 \mathrm{~Hz}), 74.1,45.3$, 27.6. IR $\left(\mathrm{CH}_{2} \mathrm{Cl}_{2}\right.$ film): 1601, 1578, 1483, 1389, 1377, 1271, 1258, 1215, 1078, 1032, 999, 918, 897, 860, $820 \mathrm{~cm}^{-1}$. HRMS (TOF-ES+) m/z: $\left[\mathrm{M}-\mathrm{H}_{2} \mathrm{O}+\mathrm{H}\right]+$ calcd for $\mathrm{C}_{11} \mathrm{H}_{11} \mathrm{ClF}$ 197.0533; found 197.0540.

1,1,1-trifluoro-2-phenylpent-4-en-2-ol (31): Prepared according to General Procedure B. Purified by column chromatography (Ethyl Acetate $/$ Hexane $=15: 85$ ) to give the product (yield: $76 \%, 164 \mathrm{mg}$ ) as a clear oil. ${ }^{1} \mathrm{H}$ NMR $\left(500 \mathrm{MHz}, \mathrm{CDCl}_{3}\right) \delta 7.58(\mathrm{~d}, J=7.7 \mathrm{~Hz}, 2 \mathrm{H})$, $7.38(\mathrm{~m}, 3 \mathrm{H}), 5.56(\mathrm{td}, J=17.0,7.7 \mathrm{~Hz}, 1 \mathrm{H}), 5.27-5.17(\mathrm{~m}, 2 \mathrm{H})$, $2.98(\mathrm{dd}, J=14.3,6.5 \mathrm{~Hz}, 1 \mathrm{H}), 2.85(\mathrm{dd}, J=14.3,8.0 \mathrm{~Hz}, 1 \mathrm{H}), 2.68$ $(\mathrm{s}, 1 \mathrm{H}),{ }^{13} \mathrm{C}\{1 \mathrm{H}\}$ NMR $\left(126 \mathrm{MHz}, \mathrm{CDCl}_{3}\right) \delta 137.0,130.5,128.7$, $128.5,126.6$ (d, $J=1.3 \mathrm{~Hz}), 124.3,122.1,75.9$ (q, $J=30.0 \mathrm{~Hz}), 40.4$ (d, $J=0.7 \mathrm{~Hz})$. Data is consistent with literature values. ${ }^{4 \mathrm{~b}, 21}$

2-(o-tolyl)pent-4-en-2-ol (32): Prepared according to General Procedure B. Purified by column chromatography (Ethyl Acetate/Hexane $=15: 85$ ) to give the product (yield: $78 \%, 137 \mathrm{mg}$ ) as a clear oil. Note: 32 is volatile under reduced pressure. ${ }^{1} \mathrm{H}$ NMR $\left(500 \mathrm{MHz}, \mathrm{CDCl}_{3}\right) \delta$ 7.27-7.26 (m, 1H), 6.98 (app. s, 3H), 5.49 (td, $J=17.4,7.3 \mathrm{~Hz}, 1 \mathrm{H})$ 4.99-4.93 (m, 2H), 2.69 (dd, $J=13.9,6.6 \mathrm{~Hz}, 1 \mathrm{H}), 2.42-2.37$ (m, 4H), $1.94(\mathrm{~s}, 1 \mathrm{H}), 1.44(\mathrm{~s}, 3 \mathrm{H}) .{ }^{13} \mathrm{C}\{1 \mathrm{H}\} \mathrm{NMR}\left(126 \mathrm{MHz}, \mathrm{CDCl}_{3}\right) \delta 144.6$, 135.4, 134.0, 132.7, 127.1, 126.1, 125.8, 119.4, 74.9, 46.7, 29.0, 22.6. Data is consistent with literature values. ${ }^{19}$

2-(naphthalen-2-yl)pent-4-en-2-ol (33): Prepared according to General Procedure B. Purified by column chromatography (Ethyl Acetate/Hexane $=15: 85$ ) to give the product (yield: $92 \%, 195 \mathrm{mg}$ ) as a clear oil. ${ }^{1} \mathrm{H}$ NMR $\left(500 \mathrm{MHz}, \mathrm{CDCl}_{3}\right) \delta 7.93(\mathrm{~s}, 1 \mathrm{H}), 7.86-7.83(\mathrm{~m}$ $3 \mathrm{H}), 7.56(\mathrm{dd}, J=8.6,1.1 \mathrm{~Hz}, 1 \mathrm{H}), 7.51-7.46(\mathrm{~m}, 2 \mathrm{H}), 5.64(\mathrm{~m}, 1 \mathrm{H})$ $5.15(\mathrm{dd}, J=23.2,13.6 \mathrm{~Hz}, 2 \mathrm{H}), 2.82(\mathrm{dd}, J=13.8,6.3 \mathrm{~Hz}, 1 \mathrm{H}), 2.61$ $(\mathrm{dd}, J=13.8,8.4 \mathrm{~Hz}, 1 \mathrm{H}), 2.23(\mathrm{~s}, 1 \mathrm{H}), 1.65(\mathrm{~s}, 3 \mathrm{H}) .{ }^{13} \mathrm{C}\{1 \mathrm{H}\} \mathrm{NMR}$ $\left(126 \mathrm{MHz}, \mathrm{CDCl}_{3}\right) \delta 145.1,133.7,133.3,132.4,128.3,128.0,127.6$, $126.2,125.8,123.7,123.3,119.7,73.9,48.4,30.1$. Data is consistent with literature values. ${ }^{19}$

2-(2-fluorophenyl)pent-4-en-2-ol (34): Prepared according to General Procedure B. Purified by column chromatography (Ethyl Acetate $/$ Hexane $=15: 85$ ) to give the product (yield: $84 \%, 151 \mathrm{mg}$ ) as a clear oil. Note: 34 is volatile under reduced pressure. ${ }^{1} \mathrm{H}$ NMR $(500$ $\left.\mathrm{MHz} \mathrm{CDCl}_{3}\right) \delta 7.56(\mathrm{t}, J=7.7 \mathrm{~Hz}, 1 \mathrm{H}), 7.24(\mathrm{~m}, 1 \mathrm{H}), 7.13(\mathrm{t}, J=7.5$ $\mathrm{Hz}, 1 \mathrm{H}), 7.04-7.00(\mathrm{dd}, J=12.2,8.1,1 \mathrm{H}), 5.60(\mathrm{dt}, J=16.8,9.2 \mathrm{~Hz})$ $5.13(\mathrm{~m}, 2 \mathrm{H}), 2.91(\mathrm{dd}, J=13.8,6.3 \mathrm{~Hz}, 1 \mathrm{H}), 2.57(\mathrm{dd}, J=13.8,8.5$ $\mathrm{Hz}, 1 \mathrm{H}), 2.42(\mathrm{~s}, 1 \mathrm{H}), 1.63(\mathrm{~s}, 3 \mathrm{H}) .{ }^{13} \mathrm{C}\{1 \mathrm{H}\} \mathrm{NMR}\left(126 \mathrm{MHz}, \mathrm{CDCl}_{3}\right)$ $\delta 159.5(\mathrm{~d}, J=245.7 \mathrm{~Hz}), 134.1(\mathrm{~d}, J=11.3 \mathrm{~Hz}), 133.7,128.8(\mathrm{~d}, J=$ $8.8 \mathrm{~Hz}), 127.5(\mathrm{~d}, J=5.0 \mathrm{~Hz}), 124.1(\mathrm{~d}, J=2.5 \mathrm{~Hz}), 119.6,116.0(\mathrm{~d}, J$ $=23.9 \mathrm{~Hz}), 72.8,46.7,28.4$. Data is consistent with literature values. ${ }^{22}$

2-(2-methoxyphenyl)pent-4-en-2-ol (35): Prepared according to General Procedure B. Purified by column chromatography (Ethyl Acetate $/$ Hexane $=30: 70$ ) to give the product (yield: $85 \%, 163 \mathrm{mg}$ ) as a clear oil. ${ }^{1} \mathrm{H}$ NMR $\left(500 \mathrm{MHz}, \mathrm{CDCl}_{3}\right) \delta 7.46(\mathrm{~m}, 1 \mathrm{H}), 7.16$ (app. s, $3 \mathrm{H}), 5.68(\mathrm{~m}, 1 \mathrm{H}), 5.15(\mathrm{~m}, 2 \mathrm{H}), 2.88(\mathrm{dd}, J=13.9,6.5 \mathrm{~Hz}, 1 \mathrm{H})$, 2.60-2.55 (m, 4H), $2.09(\mathrm{~s}, 1 \mathrm{H}), 1.63(\mathrm{~s}, 3 \mathrm{H}) .{ }^{13} \mathrm{C}\{1 \mathrm{H}\}$ NMR $(126$ $\left.\mathrm{MHz}, \mathrm{CDCl}_{3}\right) \delta 144.7,135.4,134.1,132.8,127.1,126.2,125.8$, 119.4, 74.9, 46.7, 29.1, 22.6. IR $\left(\mathrm{CH}_{2} \mathrm{Cl}_{2}\right.$ film): 2984, 1489, 1458, $1375,1256,1152,1094,1072,1055,997,932,914,760,727 \mathrm{~cm}^{-1}$. Note: HRMS analysis of 35 was unsuccessful, with unidentifiable fragmentation of the compound

$N$-(1-phenylbut-3-en-1-yl)benzenesulfonamide (36): Prepared according to General Procedure A. Purified by column chromatography (Ethyl Acetate/Hexane $=30: 70$ ) to give the product (yield: 69\%, 129 $\mathrm{mg})$ as white solids. ${ }^{1} \mathrm{H}$ NMR $\left(500 \mathrm{MHz}, \mathrm{CDCl}_{3}\right) \delta 7.66(\mathrm{~m}, 2 \mathrm{H}), 7.45$ $(\mathrm{t}, J=7.4 \mathrm{~Hz}, 1 \mathrm{H}), 7.33(\mathrm{t}, J=7.7 \mathrm{~Hz}, 2 \mathrm{H}), 7.16-7.15(\mathrm{~m}, 3 \mathrm{H}), 7.07-$ $7.05(\mathrm{~m}, 2 \mathrm{H}), 5.57-5.47(\mathrm{~m}, 1 \mathrm{H}), 5.08-4.99(\mathrm{~m}, 3 \mathrm{H}), 4.42(\mathrm{q}, J=6.7$ $\mathrm{Hz}, 1 \mathrm{H}), 2.52-2.42(\mathrm{~m}, 2 \mathrm{H}) .{ }^{13} \mathrm{C}\{1 \mathrm{H}\}$ NMR $\left(126 \mathrm{MHz}, \mathrm{CDCl}_{3}\right) \delta$ $140.6,140.3,133.2,132.5,128.8,128.5,127.6,127.2$, 126.7, 119.5, $57.3,42.0$. Data is consistent with literature values. ${ }^{23}$

$\mathrm{N}$-(1-phenylbut-3-en-1-yl)aniline (37): Prepared according to General Procedure A. Purified by column chromatography (Ethyl Acetate $/$ Hexane $=8: 92$ ) to give the product (yield: $46 \%, 103 \mathrm{mg}$ ) as white solids. Note: a mixture of rotamers $(5.7: 1)$ was observed in the puri- fied NMR. Only the major rotamer is reported here. ${ }^{1} \mathrm{H}$ NMR $(500$ $\left.\mathrm{MHz}, \mathrm{CDCl}_{3}\right) \delta$ 7.28-7.19 (m, 4H), 7.15-7.12 (m, 1H), 7.00-6.96 (m, $2 \mathrm{H}), 6.57-6.52(\mathrm{~m}, 1 \mathrm{H}), 6.41-6.39(\mathrm{~m}, 2 \mathrm{H}), 5.72-5.61(\mathrm{~m}, 1 \mathrm{H}), 5.11-$ $5.03(\mathrm{~m}, 2 \mathrm{H}), 4.29(\mathrm{dd}, J=8.0,5.1 \mathrm{~Hz}, 1 \mathrm{H}), 4.04($ broad s, $1 \mathrm{H}), 2.54-$ $2.47(\mathrm{~m}, 1 \mathrm{H}), 2.43-2.36(\mathrm{~m}, 1 \mathrm{H}) .{ }^{13} \mathrm{C}\{1 \mathrm{H}\} \mathrm{NMR}\left(126 \mathrm{MHz}, \mathrm{CDCl}_{3}\right) \delta$ 147.4, 143.7, 134.8, 129.2, 128.7, 127.1, 126.4, 118.4, 117.5, 113.6, 57.2, 43.4. Data is consistent with literature values. ${ }^{24}$

2-phenylpent-4-yn-2-ol (40a) and 2-phenylpenta-3,4-dien-2-ol (40b): Prepared according to General Procedure B with propargyl bromide ( $1.0 \mathrm{mmol}, 167 \mu \mathrm{L}, 80 \% \mathrm{wt}$ in $\mathrm{PhMe}$ ). The reaction mixture was milled for $6 \mathrm{~h}$, and then purified by column chromatography (Ethyl Acetate/Hexane $=15: 85$ ) to give an inseparable mixture of 40a and $40 \mathrm{~b}(\mathrm{a}: \mathrm{b}=4.2: 1$, yield: $71 \%, 114 \mathrm{mg})$ as a clear oil. Note: Product mixture 40 is volatile under reduced pressure. ${ }^{1} \mathrm{H}$ NMR $(500 \mathrm{MHz}$, $\left.\mathrm{CDCl}_{3}\right) \delta$ 7.42-7.39 (m, 2H, a and b), 7.28-7.24 (m, 1H, a and b), 7.19-7.15 (m, 2H, a and b), $5.47(\mathrm{t}, J=6.6 \mathrm{~Hz}, 1 \mathrm{H}, \mathrm{b}), 4.90-4.83(\mathrm{~m}$, $1 \mathrm{H}, \mathrm{b}), 2.64$ (dq, $J=16.7,1.8 \mathrm{~Hz}, 2 \mathrm{H}, \mathrm{a}), 2.41(\mathrm{~s}, 1 \mathrm{H}, \mathrm{a}), 2.19$ (s, $1 \mathrm{H}$, b), $1.96(\mathrm{~s}, 1 \mathrm{H}, \mathrm{a}), 1.57(\mathrm{~s}, 3 \mathrm{H}, \mathrm{b}), 1.55(\mathrm{~s}, 3 \mathrm{H}, \mathrm{a}) .{ }^{13} \mathrm{C}\{1 \mathrm{H}\} \operatorname{NMR}(126$ $\left.\mathrm{MHz}, \mathrm{CDCl}_{3}\right) \delta 206.0,171.3,147.2,146.4,128.3,128.3,127.2$, $127.1,125.0,124.8,100.3,80.5,79.2,73.3,73.1,71.8,60.5,34.7$, $30.5,21.1,14.3$. Data is consistent with literature values. ${ }^{25,26}$

3-methyl-2-phenyl-4 $\lambda^{5}$-penta-3,4-dien-2-ol (42): Prepared according to General Procedure B with 1-bromobut-2-yne $(1.0 \mathrm{mmol}, 88 \mu \mathrm{L})$. The reaction mixture was milled for $6 \mathrm{~h}$, and then purified by column chromatography (Ethyl Acetate/Hexane $=20: 80$ ) to give the product 42 (yield: $69 \%, 120 \mathrm{mg}$ ). Note: This product is volatile under reduced pressure. ${ }^{1} \mathrm{H}$ NMR $\left(500 \mathrm{MHz}, \mathrm{CDCl}_{3}\right) \delta 7.47(\mathrm{~d}, J=8.0 \mathrm{~Hz}, 2 \mathrm{H}), 7.34$ $(\mathrm{t}, J=7.5 \mathrm{~Hz}, 2 \mathrm{H}), 7.27-7.24(\mathrm{~m}, 1 \mathrm{H}), 4.91-4.86(\mathrm{~m}, 2 \mathrm{H}), 2.01(\mathrm{~s}$, $1 \mathrm{H}), 1.67(\mathrm{~s}, 3 \mathrm{H}), 1.57(\mathrm{~d}, J=2.7 \mathrm{~Hz}, 3 \mathrm{H}) .{ }^{13} \mathrm{C}\{1 \mathrm{H}\} \mathrm{NMR}(126 \mathrm{MHz}$, $\left.\mathrm{CDCl}_{3}\right) \delta 205.3,146.1,128.2,127.0,125.4,106.0,77.2,75.1,30.3$, 14.8. Data is consistent with literature values. ${ }^{27}$

Scale Up Reaction: The reaction was carried out using acetophenone (1.5 g, $1.46 \mathrm{~mL}, 12.5 \mathrm{mmol}, 1.0$ equiv.) according to General Procedure B. The reaction mixture was milled for $6 \mathrm{~h}$ in a $25 \mathrm{~mL}$ jar. Purified by Kugelrohr distillation to give the product (yield: $75 \%$, $1.52 \mathrm{~g}$ ) as a clear oil. The characterisation data of the product was in accordance with 20 .

\section{ASSOCIATED CONTENT}

\section{Supporting Information}

The Supporting Information is available free of charge on the ACS Publications website at DOI: XXXX including assessment of different zinc sources and the spectral data (PDF)

\section{AUTHOR INFORMATION}

\section{Corresponding Author}

*E-mail: duncan.browne@ucl.ac.uk

ORCID

Duncan L. Browne: 0000-0002-8604-229X

Ian A. Fallis: 0000-0001-7361-0182

\section{Notes}

The authors declare no competing financial interest. Information about the data that underpins the results presented in this article, including how to access them, can be found in the Cardiff University data catalogue at http://doi.org/XXXX (accessed XXXX).

\section{ACKNOWLEDGMENTS}

We gratefully acknowledge the School of Chemistry, Cardiff University for generous support, I.A.F. and D.L.B. are grateful to the European Union, Tenovus Cancer Charity, the National Research Network Wales and Cardiff University for a Knowledge Economy and Skills Schollorship (KESS2) to R.S. and Fellowship to J.Y. 


\section{REFERENCES}

(1) a) Lumbroso, A.; Cooke, M. L.; Breit, B. Catalytic Asymmetric Synthesis of Allylic Alcohols and Derivatives and their Applications in Organic Synthesis. Angew. Chem. Int. Ed. 2013, 52, 1890-1932. b) Blomberg, C.; Hartog, F. A. The Barbier Reaction - A One-Step Alternative for Syntheses via Organomagnesium Compounds. Synthesis 1977, 18-30.

(2) Barbier, P. C. R., Synthèse du dimethylheptenol. Hebd. Séances Acad. Sci. 1899, 128, 110-111.

(3) For examples of Barbier (-type) reaction processes using different metals see; Zn: a) Zhao, L. M.; Gao, H. S.; Li, D. F.; Dong, J.; Sang, L. L.; Ji, J. Zinc-mediated $\alpha$-regioselective Barbier-type cinnamylation reactions of aldehydes, ketones and esters. Org. Biomol. Chem. 2017, 15, 4359-4366. Mg: b) Kopach, M. E.; Roberts, D.; Johnson, M. D.; Groh, J. M.; Adler, J. J.; Schafer, J. P.; Kobierski, M. E.; Trankle, W. G. Magnesium-Mediated Carbon-Carbon Bond Formation in Aqueous Media: Barbier-Grignard Allylation and Pinacol Coupling of Aldehydes. Green Chem. 2012, 14, 1524-1536. Sm: c) Basu, M. K.; Banik, B. K. Samarium-mediated Barbier reaction of carbonyl compounds. Tetrahedron Lett. 2001, 42, 187-189. Al: d) Yuan, S. Z.; Liu, J. Nano-Aluminum Powder Mediated Allylation of Carbonyl Compounds in Aqueous Media. Chin. J. Chem. 2008, 26, 804-806. In: e) Auge, J.; Germain, N. L.; Marque, S.; Seghrouchni, L. Indium-catalyzed Barbier allylation reaction. J. Organomet. Chem. 2003, 679, 79-83. Cd: f) Zhou, C. L.; Wang, Z. Y. CadmiumMediated Carbonyl Benzylation in Tap Water. Synthesis 2005, 10, 1649-1655. Sb: g) Ren, P. D.; Jin, Q. H.; Yao, Z. P. Barbier-Type Allylation of Aldehydes with Active Metallic Antimony. Synth. Commun. 1997, 27, 2761-2767. Pb: h) Tanaka, H.; Hamatant, T.; Yamashita, S.; Torii, S. Lead Promoted Barbier-Type Reaction of Propargyl Bromide with Aldehydes. Chem. Lett. 1986, 1461-1462. Sn: i) Wang, Z. Y.; Zha, Z. G.; Zhou, C. L. Application of Tin and Nanometer Tin in Allylation of Carbonyl Compounds in Tap Water. Org. Lett. 2002, 4, 1683-1685. Bi: j) Wada, S.; Hayashi, N.; Suzuki, $\mathrm{H}$. Noticeable facilitation of the bismuth-mediated Barbier-type allylation of aromatic carbonyl compounds under solvent-free conditions. Org. Biomol. Chem. 2003, 1, 2160-2163. Mn: k) Cahiez, G.; Chavant, P. Y. Organomanganese (II) reagents XX: Manganese mediated Barbier and Reformatsky like reactions an efficient route to homoallylic alcohols and $\beta$-acetoxyesters. Tetrahedron Lett. 1989, 30, 7373-7376.

(4) a) Nakamura, S.; Hara, Y.; Furukawa, T.; Hirashita, T. Enantioselective Barbier-type allylation of ketones using allyl halide and indium in water. $R S C A d v$. 2017, 7, 15582-15585. b) Haddad, T. D.; Hirayama, L. C.; Singaram, B. Indium-Mediated Asymmetric Barbier-Type Allylations: Additions to Aldehydes and Ketones and Mechanistic Investigation of the Organoindium Reagents. J. Org. Chem. 2010, 75, 3, 642-649.

(5) For overviews of aqueous Barbier-type reactions see: a) Li, C. J. Organic reactions in aqueous media - with a focus on carboncarbon bond formation. Chem. Rev. 1993, 93, 2023-2035 and references cited therein. b) Li, C. J., Developing metal-mediated and catalyzed reactions in air and water. Green Chem. 2002, 4, 1-4 and references cited therein. c) Shen, Z. L.; Loh, T. P. Indium-CopperMediated Barbier-Grignard-Type Alkylation Reaction of Imines in Aqueous Media. Org. Lett. 2007, 9, 5413-5416 and references cited therein. d) Shen, Z.-L.; Cheong, H.-L.; Loh, T.-P. Indium/coppermediated conjugate addition of unactivated alkyl iodides to $\alpha, \beta$ unsaturated carbonyl compounds in water. Tetrahedron Lett. 2009, 50, 1051-1054 and references cited therein.

(6) For example protocols concerning the preparation of activated zinc see: a) Rieke, R. D.; Uhm, S. J.; Hudnall, P. M. Activated metals. Preparation of highly reactive zinc. J. Chem. Soc., Chem. Commun. 1973, 269b-270. b) Rieke, R. D.; Li, P. T.-J.; Burns, T. P.; Uhm, S. T. Preparation of highly reactive metal powders. New procedure for the preparation of highly reactive zinc and magnesium metal powders. $J$. Org. Chem. 1981, 46, 4323-4324. c) Guijarro, A.; Rosenberg, D. M.; Rieke, R. D. The Reaction of Active Zinc with Organic Bromides. $J$. Am. Chem. Soc. 1999, 121, 4155-4167.d) Berk, S. C.; Knochel, P.; Yeh, M. C. P. General approach to highly functionalized benzylic organometallics of zinc and copper. J. Org. Chem. 1988, 53, 5789-
5791. e) Picotin, G.; Miginiac, P. Activation of zinc by trimethylchlorosilane. An improved procedure for the preparation of betahydroxy esters from ethyl bromoacetate and aldehydes or ketones (Reformatsky reaction). J. Org. Chem. 1987, 52, 4796-4798. f) Ikegami, R.; Koresawa, A.; Shibata, T.; Takagi, K. Functionalized Arylzinc Compounds in Ethereal Solvent: Direct Synthesis from Aryl Iodides and Zinc Powder and Application to Pd-Catalyzed Reaction with Allylic Halides. J. Org. Chem. 2003, 68, 2195-2199. g) Huo, S. Highly Efficient, General Procedure for the Preparation of Alkylzinc Reagents from Unactivated Alkyl Bromides and Chlorides. Org. Lett. 2003, 5, 423-425. h) Kimura, M.; Seki, M. A novel procedure for the preparation of zinc reagents: a practical synthesis of (+)-biotin. Tetrahedron Lett. 2004, 45, 1635-1637.

(7) For some recent reviews on mechanochemistry see: a) Howard, J. L.; Cao, Q.; Browne, D. L. Mechanochemistry as an emerging tool for molecular synthesis: what can it offer? Chem. Sci. 2018, 9, 30803094.

b) Andersen, J.; Mack, J. Mechanochemistry and organic synthesis: from mystical to practical. Green Chem. 2018, 20, 1435-1443. c) Friscic, T.; Halasz, I.; Strukil, V.; Eckert-Maksic, M.; Dinnebier, R. E. Mechanochemistry: A Force of Synthesis. ACS Cent. Sci. 2017, 3, 13-19. d) Hernandez, J. G.; Bolm, C.,Altering Product Selectivity by Mechanochemistry. J. Org. Chem. 2017, 82, 4007-4019. e) Eguaogie, O.; Vyle, J. S.; Conlon, P. F.; Gilea, M. A.; Liang, Y. Mechanochemistry of nucleosides, nucleotides and related materials. Beilstein $J$. Org. Chem. 2018, 14, 955-970. f) Metro, T.-X.; Martinez, J.; Lamaty, F. 1,1'-Carbonyldiimidazole and Mechanochemistry: A Shining Green Combination. ACS Sustainable Chem. Eng. 2017, 5, 95999602. g) Tan, D.; Garcia, F. Main group mechanochemistry: from curiosity to established protocols. Chem. Soc. Rev. 2019, 48, 22742292.

(8) a) Cao, Q.; Howard, J. L.; Wheatley, E.; Browne, D. L. Mechanochemical Activation of Zinc and Application to Negishi CrossCoupling. Angew. Chem. Int. Ed. 2018, 57, 11339-11343. b) Cao, Q.; Stark, R. T.; Fallis, I. A.; Browne, D. L. A Ball-Milling-Enabled Reformatsky Reaction. ChemSusChem. 2019, 12, 2554-2557. c) Liu, H.-W.; Xu, H.; Shao, G.; Wang, G.-W. Zinc-Mediated Reductive Cyclization of [60]Fullerene with Enones and Subsequent Dehydration under Solvent-Free and Ball-Milling Conditions. Org. Lett. 2019, $21,2625-2628$

(9) For some examples of mechanochemical reactions that appear to have reduced sensitivity to water/aerobic conditions see ref $18+$ a) Waddell, D. C.; Clark, T. D.; Mack, J. Conducting moisture sensitive reactions under mechanochemical conditions. Tetrahedron Lett. 2012, 53, 4510-4513. b) Shao, Q.-L.; Jiang, Z.-J,; Su, W.-K. Solvent-free mechanochemical Buchwald-Hartwig amination of aryl chlorides without inert gas protection. Tetrahedron Lett. 2018, 59, 2277-2280. c) Cao, Q.; Nicholson, W. I.; Jones, A. C.; Browne, D. L. Robust Buchwald-Hartwig amination enabled by ball-milling. Org. Biomol. Chem. 2019, 17, 1722-1726. d) Seo, T.; Ishiyama, T.; Kubota, K.; Ito, H. Solid-state Suzuki-Miyaura cross-coupling reactions: olefinaccelerated $\mathrm{C}-\mathrm{C}$ coupling using mechanochemistry. Chem. Sci. 2019, $10,8202-8210$

(10) a) Friščić, T.; Trask, A. V, Jones, W.; Motherwell, W. D. S. Screening for Inclusion Compounds and Systematic Construction of Three-Component Solids by Liquid-Assisted Grinding. Angew. Chem. Int. Ed. 2006, 45, 7546-7550. b) Friščić, T.; Childs, S. L.; Rizvi, S. A. A.; Jones, W. The role of solvent in mechanochemical and sonochemical cocrystal formation: a solubility-based approach for predicting cocrystallisation outcome. CrystEngComm 2009, 11, 418-426. c) Hasa, D.; Schneider, R. G.; Voinovich, D.; Jones, W. Cocrystal Formation through Mechanochemistry: from Neat and Liquid-Assisted Grinding to Polymer-Assisted Grinding. Angew. Chem. Int. Ed. 2015, 54,7371-7375.

(11) For some examples of Liquid-Assisted Grinding applied to cocrystal formation see: a) Karki, S.; Friščić, T.; Jones, W.; Motherwell, W. D. S. Screening for Pharmaceutical Cocrystal Hydrates via Neat and Liquid-Assisted Grinding. Mol. Pharmaceutics 2007, 4, 347-354. b) Friščić, T.; Fabian, L.; Burley, J. C.; Jones, W.; Motherwell, W. D. $\mathrm{S}$. Exploring cocrystal-cocrystal reactivity via liquid-assisted grinding: the assembling of racemic and dismantling of enantiomeric cocrystals. Chem. Commun. 2006, 48, 5009-5011. c) Fernandes, J. A.; 
Sardo, M.; Marfa, L.; Choquesillo-Lazarte, D.; Masciocchi, N. X-ray and NMR Crystallography Studies of Novel Theophylline Cocrystals Prepared by Liquid Assisted Grinding. Cryst. Growth Des. 2015, 15, 3674-3683. d) Jung, S.; Choi, I.; Kim, I. W. Liquid-Assisted Grinding to Prepare a Cocrystal of Adefovir Dipivoxil Thermodynamically Less Stable than Its Neat Phase. Crystals, 2015, 5, 583-591. e) Mukherjee, A.; Rogers, R. D.; Myerson, A. S. Cocrystal formation by ionic liquid-assisted grinding: case study with cocrystals of caffeine. CrystEngComm 2018, 20, 3817-3821. f) Belenguer, A. M.; Lampronti, G. I.; De Mitri, N.; Driver, M.; Hunter, C. A.; Sanders, J. K. M. Understanding the Influence of Surface Solvation and Structure on Polymorph Stability: A Combined Mechanochemical and Theoretical Approach. J. Am. Chem. Soc. 2018, 140, 17051-17059.

(12) For some examples of Liquid-Assisted Grinding applied to covalent bond formation see: a) Chen, L.; Regan, M.; Mack, J. The Choice Is Yours: Using Liquid-Assisted Grinding To Choose between Products in the Palladium-Catalyzed Dimerization of Terminal Alkynes. ACS Catal. 2016, 6, 868-872. b) Howard, J. L.; Sagatov, Y.; Repusseau, L.; Schotten, C.; Browne, D. L. Controlling reactivity through liquid assisted grinding: the curious case of mechanochemical fluorination. Green Chem. 2017, 19, 2798-2802. c) Howard, J. L.; Sagatov, Y.; Browne, D. L. Mechanochemical electrophilic fluorination of liquid beta-ketoesters. Tetrahedron 2018, 74, 3118-3123. d) Jiang, Z. J.; Li, Z. H.; Yu, J. B.; Su, W. K. Liquid-Assisted Grinding Accelerating: Suzuki-Miyaura Reaction of Aryl Chlorides under High-Speed Ball-Milling Conditions. J. Org. Chem. 2016, 81, 1004910055. e) Howard, J. L.; Brand, M. C.; Browne, D. L. Switching Chemoselectivity: Using Mechanochemistry to Alter Reaction Kinetics. Angew. Chem. Int. Ed. 2018, 57, 16104-16108.

(13) Lichtenberg, C.; Engel, J.; Spaniol, T. P.; Englert, U.; Raabe, G.; Okuda, J. Bis(allyl)zinc Revisited: Sigma versus Pi Bonding of Allyl Coordination. J. Am. Chem. Soc. 2012, 134, 9805-9811.

(14) Denmark, S. E.; Nguyen, S. T. Catalytic, Nucleophilic Allylation of Aldehydes with Allyl Acetate. Org. Lett. 2009, 11, 781-784.

(15) Li. G.; Zhao, G. Allylation of Aldehydes and Imines: Promoted by Reuseable Polymer-Supported Sulfonamide of N-Glycine. Org. Lett. 2006, 8, 633-636.

(16) Lin, M.-H.; Hung, S.-F.; Lin, L.-Z.; Tsai, W.-S.; Chuang, T.-H. Tin Mediated Allylation Reactions of Enol Ethers in Water. Org. Lett. 2011, 13, 332-335.
(17) Sio, V. D.; Massa, A.; Scettri. A. Chiral sulfoxides as activators of allyl trichlorosilanes in the stereoselective allylation of aldehydes. Org. Biomol. Chem. 2010, 8, 3055-3059.

(18) Heathcock, C. H.; Kiyooka, S.; Blumenkopf, T. A. Acyclic stereoselection. 22. Diastereofacial selectivity in the Lewis acid mediated reactions of allylsilanes with chiral aldehydes and enones. J. Org. Chem. 1984, 49, 4214-4223.

(19) Shen, K.-H.; Yao, C.-F. Novel and Efficient Method for the Allylation of Carbonyl Compounds and Imines Using Triallylaluminum. J. Org. Chem. 2006, 71, 3980-3983.

(20) Cunningham, A.; Mokal-Parech, V.; Wilson, C.; Woodward, S. On the use of mixtures of organotin species for catalytic enantioselective ketone allylation - a detective story. Org. Biomol. Chem. 2004, 2, 741-748.

(21) Felix, C.; Laurent, A.; Mison, P. Mise en évidence d'une réactivité spécifique des trifluorométhylcétones vis-à-vis du bromure d'allylmagnésium. J. Fluor. Chem. 1995, 70, 71-82.

(22) Fandrick, K. R.; Fandrick, D. R.; Gao, J. J.; Reeves, J. T.; Tan, Z.; Li, W.; Song, J.; Lu, B.; Yee, N. K.; Senanayake, C. H. Mild and General Zinc-Alkoxide-Catalyzed Allylations of Ketones with Allyl Pinacol Boronates. Org. Lett. 2010, 12, 3748-3751.

(23) Moriyama, K.; Kuramochi, M.; Fuji, K.; Morita, T.; Togo, H. Nitroxyl-Radical-Catalyzed Oxidative Coupling of Amides with Silylated Nucleophiles through N-Halogenation. Angew. Chem. Int. Ed. 2016, 55, 14546-14551.

(24) Fan, R.; Pu, D.; Qin, L.; Wen, F.; Yao, G.; Wu, J. Efficient Three-Component One-Pot Benzylation and Allylation of Aldehydes and Amines for Synthesis of Homobenzylamines and Homoallylamines. J. Org. Chem. 2007, 72, 3149-3151.

(25) Umaña, C. A.; Cabezas, J. A. Palladium-Catalyzed One-Pot Conversion of Aldehydes and Ketones into 4-Substituted Homopropargyl Alcohols and 5-En-3-yn-1-ols. J. Org. Chem. 2017, 82, 95059514.

(26) Luo, H.; Ma, S. CuI-Catalyzed Synthesis of Functionalized Terminal Allenes from 1-Alkynes. Eur. J. Org. Chem. 2013, 30413048.

(27) Sai, M.; Yorimitsu, H.; Oshima, K., Allyl-, Allenyl-, and Propargyl-Transfer Reactions through Cleavage of C-C Bonds Catalyzed by an N-Heterocyclic Carbene/Copper Complex: Synthesis of Multisubstituted Pyrroles. Angew. Chem. Int. Ed. 2011, 50, 3294-3298. 